\title{
Infezioni urinarie nel trapiantato renale
}

\author{
J. Romagnoli, E. Favi, M.P. Salerno, F. Citterio
}

Dipartimento di Scienze Chirurgiche, Istituto di Clinica Chirurgica, Università Cattolica del Sacro Cuore, Policlinico Gemelli, Roma

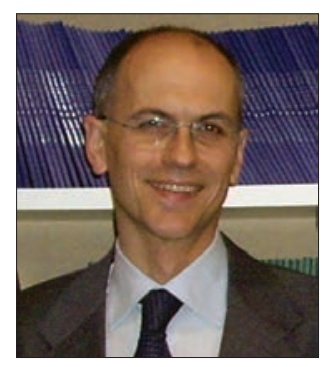

Jacopo Romagnoli

\section{Introduzione}

Le infezioni delle vie urinarie (IVU) sono il tipo di infezione più frequente nei riceventi un trapianto di rene (1-3), con un'incidenza variabile tra il 6\% e l' $86 \%$ a seconda delle casistiche (4-6) e sono ancora oggi responsabili di notevole morbilità e di disfunzione del graft. La notevole variabilità nell'incidenza di IVU riportata in letteratura è verosimilmente legata a più fattori: definizioni e criteri diagnostici non univoci, strategie antibiotiche diverse a seconda delle abitudini locali, resistenze microbiche legate a differenze geografiche.

La maggior incidenza di IVU si registra nel primo anno post-trapianto (74\%), in particolare nel primo trimestre, per poi decrescere nei mesi successivi, fino a raggiungere un plateau (22\%) a 3 anni dal trapianto (7). Un importante studio retrospettivo su 28.942 pazienti ha dimostrato come le IVU contratte tardivamente, sebbene meno frequenti rispetto allimmediato post-trapianto, si associno a un considerevole incremento di mortalità e perdita del graft (8). Nonostante l'argomento IVU nel trapiantato sia di notevole interesse clinico, la carenza di studi basati sull'evidenza contribuisce a mantenere una situazione di scarsa chiarezza su quale sia l'approccio migliore alla gestione di questa complicanza.

\section{Definizioni}

Con il termine batteriuria si definisce la presenza di batteri nelle urine, per fare diagnosi nel paziente asintomatico sono necessarie almeno 100.000 unità formanti colonie $(\mathrm{CFU}) / \mathrm{mL}$, nel paziente sintomatico è invece sufficiente dimostrare la presenza di $100 \mathrm{CFU} / \mathrm{mL}$. Aggregati fungini con concentrazione di unità formanti co- lonie $>$ di $10^{5} / \mathrm{mL}$ definiscono la funguria (9).

Per IVU ricorrente si intende una infezione sintomatica che compare dopo la risoluzione di un precedente episodio di IVU, nel caso specifico si tratta in genere di tre o più episodi sintomatici nell'arco di 12 mesi. L'infezione ricorrente può a sua volta essere distinta in reinfezione 0 ricaduta: nel primo caso si tratta di un nuovo episodio infettivo che si manifesta dopo l'avvenuta negativizzazione della batteriuria nell'urinocoltura, ed è generalmente sostenuta da un ceppo diverso; nel caso della ricaduta l'infezione è sostenuta dallo stesso ceppo e si manifesta solitamente entro 2 settimane dalla sospensione del trattamento antibiotico.

\section{Fattori di rischio}

Nel trapiantato di rene alcuni fattori di rischio per IVU sono comuni a quelli della popolazione generale, ad esempio l'età avanzata $(6,8,11)$, il sesso femminile $(6$, $8,10,11)$ in relazione a motivi sia anatomici - maggiore brevità dell'uretra, minore distanza tra ano e orifizio uretrale - sia ormonali - ridotta secrezione di estrogeni nel periodo post-menopausale determinante alterazioni del $\mathrm{pH}$ e della flora vaginale (12), la presenza di litiasi, alterazioni anatomiche o funzionali delle vie urinarie determinanti stasi urinaria o reflusso $(4,5)$. Così pure altre condizioni mediche, quali il diabete (13) e la stessa insufficienza renale, che consentono ai germi di bypassare con maggiore facilità le difese immunitarie dell'ospite.

Tuttavia, vi sono fattori caratteristici legati al trapianto renale che rendono il ricevente maggiormente vulnerabile. La presenza del catetere vescicale, che nella popolazione generale aumenta il rischio di batteriuria del $5 \%$ ogni giorno, comporta verosimilmente un aumento del rischio nel trapiantato, anche se non vi sono in letteratura studi specifici su questa popolazione (5). La ri- 
mozione precoce del catetere vescicale, dalla seconda giornata post-trapianto, sembrerebbe favorire infatti una riduzione nell'incidenza di IVU (4).

Alcune condizioni patologiche sia dei reni nativi sia del rene trapiantato, predispongono allo sviluppo di focolai infiammatori o infettivi (ad esempio, malattia policistica, litiasi, pielonefrite, reflusso vescico-ureterale), analogamente gli esiti chirurgici di alcuni interventi (ad esempio, monconi chirurgici di uretere a seguito di nefrectomia di reni nativi) possono agire da reservoire per patogeni, tali focolai possono agire come delle vere e proprie bombe a orologeria in un paziente immunodepresso, la loro importanza non deve essere sottostimata nel ragionamento diagnostico.

L'uso di immunosoppressori, in particolare la dose e la combinazione di farmaci, tra cui ad esempio gli antimetaboliti, incrementa il rischio di infezioni in generale e quindi anche quello di IVU (3). Il rischio è maggiore nel primo anno post-trapianto, quando il livello di immunosoppressione è più elevato. Tuttavia, anche in seguito, un evento quale ad esempio un episodio di rigetto può elevare nuovamente il livello di rischio in seguito all'incremento della terapia immunosoppressiva. Il rigetto acuto si associa infatti a un aumento dell'incidenza di IVU, specialmente se il paziente ha ricevuto anticorpi policlonali, ad esempio timoglobuline (2).

Manovre strumentali sull'apparato urinario (ad esempio, cistoscopia eseguita per ematuria) possono incrementare notevolmente il rischio di infezione, il trauma uroteliale prodotto determina infatti una perdita di glicosaminoglicani dalla superficie uroteliale e introduce patogeni nelle cellule uroteliali e nel biofilm che ricopre il catetere vescicale o lo stent, ponendo le basi per lo sviluppo di IVU ricorrenti (4). Analogamente, la presenza di stent ureterovescicale, posizionato per prevenire il leak anastomotico, si associa a un aumento del rischio di 1,5 volte (14).

Probabilmente anche la manipolazione del rene e dell'uretere durante l'intervento chirurgico predispongono allo sviluppo di IVU (5), anche se mancano evidenze sufficienti in letteratura a supporto di questa teoria. Altrettanto probabile è la relazione tra contaminazione del liquido di perfusione/conservazione dell'organo e sviluppo di IVU nell'immediato post-trapianto, è buona norma a questo proposito inviare routinariamente per coltura un campione di liquido prelevato al momento dell'estrazione del rene dal suo contenitore.

Il trapianto da donatore vivente sembra invece essere associato a una incidenza inferiore di IVU rispetto al trapianto da donatore cadavere, probabilmente in rapporto a inferiori tempi di ischemia fredda, minore danno da ischemia-riperfusione, minore incidenza di ritardata ripresa funzionale del graft (2).

\section{Microorganismi responsabili di UTI}

La maggior parte delle IVU nel trapiantato è causata da batteri gram negativi e da enterococchi. I germi di più comune riscontro sono l'Escherichia coli (E. coli), isolato nel $70-95 \%$ dei casi (12), seguito dagli enteroccocchi nel 5-20\% dei casi. Altri patogeni occasionali includono il Proteus mirabilis e la Klebsiella nelle sue varie specie $(15,16)$. Pseudomonas aeruginosa, stafilococchi coagulasi-negativi ed Enterobacter cloacae sono generalmente causa di infezioni nell'immediato post-trapianto, mentre le specie predominanti nel lungo termine sono l'E. coli e l'Enterococco (5).

Le infezioni nel paziente trapiantato sono spesso polimicrobiche e sostenute da germi multiresistenti, nel $50 \%$ dei casi il principale responsabile è un ceppo multi resistente di E. coli, insieme con l'Enterobacter faecalis (15\%) e una eterogenea popolazione microbica rappresentata da Serratia, Pseudomonas, Providencia e stafilococco Epidermidis $(16,17)$. Secondo uno studio basato su infezioni comunitarie l'E. coli è responsabile anche del $78 \%$ delle forme ricorrenti (18), la sua notevole patogenicità uroteliale sarebbe determinata dall'espressione delle fimbrie di tipo P o 1 (19), che garantiscono al germe la capacità di aderire tenacemente all'urotelio dell'ospite. Nei pazienti con lunga storia di dialisi e ripetute ospedalizzazioni non è infrequente invece la presenza di stafilococchi meticillino resistenti (MRSA) ed enterococchi vancomicino resistenti (VRE) (20).

Tra i germi responsabili di IVU nel post-trapianto si possono incontrare anche funghi, virus, parassiti o micoplasmi. Le IVU fungine colpiscono maggiormente i riceventi affetti da diabete, la Candida è il fungo più spesso responsabile. Tipicamente si tratta di infezioni opportunistiche in pazienti fortemente immunodepressi, spesso già affetti da altre forme infettive in trattamento antibiotico o ricoverati in unità di terapia intensiva. Sono infezioni assai difficili da trattare e la loro gestione è resa tra l'altro complicata dall'interferenza di molti antifungini con il metabolismo dei principali immunosoppressori.

Altro patogeno emergente è il BK virus, appartenente alla famiglia dei poliomavirus, responsabile di una infezione latente che tende a riattivarsi in corso di immunosoppressione. Circa il 70\% dei soggetti adulti ne è portatore, fattori di rischio per lo sviluppo dell'infezione sono la sieropositività del donatore, il grado di immunosoppressione, l'uso di tacrolimus, il rigetto (20). È responsabile di nefrite tubulointerstiziale del graft e stenosi ureterale, può portare a perdita del graft nel $45 \%$ dei casi (21). La diagnosi viene fatta mediante dosaggio del DNA virale nel sangue e nelle urine e ricerca delle de- 
coy cell nelle urine, tuttavia per la conferma definitiva è necessaria la biopsia del graft, che dimostra la presenza degli inclusi virali. Non esiste al momento alcuna terapia specifica convalidata, sebbene alcuni risultati positivi siano stati raggiunti con l'uso della Leflunomide, che associa azione antivirale e immunosoppressiva e non è nefrotossica (20).

\section{Le infezioni urinarie ricorrenti}

Non esistono dati certi sulla reale incidenza di infezioni ricorrenti nei trapiantati di rene. Nella popolazione generale colpiscono maggiormente il sesso femminile (12), circa il $25 \%$ delle pazienti che hanno avuto un primo episodio di IVU sviluppano prima o poi una infezione ricorrente (22) ed è ragionevole pensare che nella popolazione trapiantata tale incidenza sia sovrapponibile, se non addirittura maggiore. Il fattore di rischio più comune nelle giovani donne nel periodo premenopausale è il rapporto sessuale, mentre in menopausa acquiscono maggior importanza il deficit di estrogeni, l'incontinenza, la presenza di cistocele, la storia di chirurgia genitourinaria, un elevato residuo post-minzionale, diverticoli vescicali e pregresse IVU. Il germe più frequentemente isolato è $l ' E$. coli. È stato dimostrato che le pazienti con IVU ricorrenti sono soggette a una più facile colonizzazione vaginale da parte di vari uropatogeni, $E$. coli in primis, seguito da altri batteri gram negativi. I ceppi di $E$. coli responsabili di IVU ricorrenti sono capaci di produrre una capsula di polisaccaridi acidi che ha il duplice effetto di proteggerli dalla fagocitosi da parte dei leucociti polimorfonucleati e di inibire l'attivazione del complemento (23). Essi tendono a colonizzare la mucosa periuretrale per poi migrare all'interno della vescica, dove producono l'esfoliazione degli strati superficiali dell'urotelio per poi annidarsi negli strati più profondi, da dove poi riemergono periodicamente riaccendendo l'infezione (24). Verosimilmente tale caratteristica giustifica la frequente difficoltà nell'eradicazione dell'infezione in pazienti immunodepresse. Se nel maschio le IVU hanno un incidenza minore, in relazione alla maggiore lunghezza dell'uretra e alla presenza di sostanze ad azione antibatterica nel secreto prostatico, sono però più frequenti dopo i 40 anni e sono spesso associate con fenomeni ostruttivi legati all'ipertrofia prostatica. Le forme più comuni sono le prostatiti batteriche e hanno una notevole tendenza alla recidiva. Sono sostenute prevalentemente da E. coli ed enterobatteriacee, talora nel soggetto anziano da Proteus, Klebsiella, Serratia, Pseudomonas ed enterococchi.

\section{Diagnosi}

Come nel paziente immunocompetente, la sintomatologia di esordio può variare a seconda della localizzazione dell'infezione, tuttavia l'identificazione precoce dell'IVU non è sempre facile, il paziente trapiantato di rene tende ad essere per definizione paucisintomatico, sia per effetto dell'immunosoppressione, che lo rende incapace di organizzare una adeguata risposta infiammatoria, sia per il fatto che il rene trapiantato è denervato.

La ricerca di una conferma al sospetto diagnostico deve essere rapida e accurata. Prevede in successione l'esame delle urine chimico-fisico e microscopico, l'urinocoltura, la determinazione dei valori ematici di creatinina, leucociti, proteina $\mathrm{C}$ reattiva, e dei farmaci immunosoppressori. In presenza di alterazione dei valori di creatinina è necessario eseguire una ecografia del rene trapiantato alla ricerca di idronefrosi, focolai infettivi parenchimali, raccolte intra o perirenali. $\mathrm{E}$ utile inoltre chiedere al radiologo di verificare la presenza di un eventuale residuo vescicale post-minzionale, indice di un problema ostruttivo.

E importante definire altezza e tipologia dellinfezione, le tecniche di radiologia tradizionale (ad esempio, $\mathrm{Rx}$ diretta addome) possono aiutare a individuare la presenza di calcoli nelle vie urinarie native o nel rene trapiantato. Tecniche di imaging più sofisticate (ad esempio, TAC con o senza MDC) sono indicate in caso di cisti renali complesse o sospetti ascessi renali. Nel sospetto di reflusso è indicata la cistografia minzionale, utile anche nel sospetto di ostruzione al deflusso di urina, insieme con le indagini di tipo urodinamico, come ad esempio la flussimetria. Quest'ultima, insieme alla cistomanometria, è di aiuto nel rivelare quadri di disfunzione vescicale. Ove si confermi un quadro ostruttivo a livello ureterale è indicato il posizionamento di una nefrostomia a scopo diagnostico (pielografia) ma anche terapeutico (stenting o ureteroplastica con pallone). Indagini invasive, come ad esempio la cistoscopia, sono giustificate per escludere la presenza di carcinoma uroepiteliale (25). Prima di qualsiasi manovra invasiva è consigliabile una profilassi antibiotica monodose con un antibiotico a largo spettro attivo sulle vie urinarie, ad esempio Ciprofloxacina.

Nei pazienti con IVU ricorrente è necessario un approfondimento diagnostico sull'apparato urinario, alla ricerca di focolai infettivi non eradicati. Deve essere esclusa la presenza di anomalie anatomiche o funzionali dell'apparato urinario, senza trascurare l'indagine dei reni nativi, che possono essere causa di infezione ricorrente in quanto sede di cisti ascessualizzate, litiasi o reflusso vescico-ureterale.

La TAC insieme con la Scintigrafia con acido dimercap- 
tosuccinico (DMSA) si sono dimostrate utili nell'identificazione di cicatrici corticali renali, espressione di patologia parenchimale renale, di frequente riscontro in pazienti con IVU ricorrenti.

Vale la pena infine ricordare che rigetto acuto del graft e IVU possono talora presentarsi in modo simile e addirittura essere sincroni, in questo caso la diagnostica deve mirare a confermare entrambi i quadri. Allo stato attuale l'unico esame in grado di discriminare in modo affidabile tra rigetto acuto e pielonefrite è la biopsia del rene trapiantato (4). La procedura comporta un modesto rischio di sanguinamento e di infezione (4) a fronte di un elevato livello di accuratezza diagnostica.

\section{Profilassi}

Nel perioperatorio è pratica comune somministrare una profilassi antibiotica monodose prima dell'induzione dell'anestesia, generalmente una cefalosporina di seconda o terza generazione, ad esempio cefazolina 10 2 grammi e.v., oppure un fluorochinolonico, più spesso ciprofloxacina $500 \mathrm{mg}$ e.v. (26).

Nel postoperatorio la gestione è molto diversa a seconda delle abitudini locali, alcuni Centri attuano la profilassi short term, aggiungendo una somministrazione postoperatoria in modo da mantenere una protezione nelle prime 24 ore dall'intervento, la maggior parte però protrae la somministrazione fino alla rimozione del catetere vescicale (4), tuttavia non vi sono in letteratura studi prospettici controllati che dimostrino una minore incidenza di infezioni con questa strategia. La profilassi antibiotica con trimetoprim-sulfametossazolo (TMPS) si è dimostrata efficace nel ridurre la frequenza di tutte le infezioni batteriche, incluse le IVU (27). L'uso di TMPS per i primi 9 mesi post-trapianto si associa con una riduzione statisticamente significativa nel numero delle infezioni batteriche, comprese le IVU (28), l'evidenza derivante da numerosi studi, e in particolar modo le linee guida KDIGO, suggeriscono infatti l'uso profilattico post-trapianto di TMPS per 6-12 mesi (28). Non vi sono tuttavia dati certi sul reale beneficio dell'uso di tale farmaco oltre i 9 mesi dal trapianto. Per i pazienti allergici a TMPS viene raccomandato l'uso alternativo di Nitrofurantoina, al fine di ridurre la resistenza agli altri agenti antibatterici comunemente usati (4).

\section{Trattamento}

Il trattamento delle IVU post-trapianto deve essere tempestivo, al fine di contenere l'impatto dell'episodio in- fettivo sulla funzione e sulla sopravvivenza del graft. La terapia è inizialmente empirica e deve mirare a coprire batteri Gram positivi e Gram negativi, successivamente deve essere mirata sulla base dell'antibiogramma, fino all'eradicazione completa del germe.

In generale, nella scelta di un antibiotico si deve tenere presente la possibilità di interazione con i comuni farmaci immunosoppressori. Un antibiotico efficace e poco tossico, può essere infatti inadeguato perché interferisce con uno dei sistemi enzimatici che entra nel metabolismo della ciclosporina o del tacrolimus (ad esempio, il citocromo $\mathrm{P} 450$ sintetasi), incrementandone o riducendone l'attività e quindi determinando fluttuazioni nei livelli ematici di tali farmaci, con conseguente rischio di rigetto (sottoesposizione al farmaco) o tossicità e infezione (sovraesposizione al farmaco). Cautela è richiesta ad esempio nell'uso dei macrolidi e degli antifungini azolici, entrambi responsabili di un incremento dei livelli ematici di ciclosporina, tacrolimus e rapamicina (20). Amoxicillina + acido clavulanico, cefalosporine e fluorochinoloni sono più sicuri e possono essere utilizzati in prima battuta nell'approccio empirico al trattamento dell'IVU. Anche TMPS viene usato spesso, va ricordato comunque che si tratta di un batteriostatico e che il suo uso in associazione con ciclosporina può determinare un aumento dei valori di creatinina, reversibile al momento della sospensione (4). La fosfomicina è un antibiotico orale che può risultare utile nel trattamento ambulatoriale di infezioni sostenute da germi Gram negativi nei confronti dei quali può avere un'efficacia talora paragonabile a quella di antibiotici somministrabili unicamente per e.v. Vale la pena ricordare che essendo il paziente "monorene" la dose di antibiotico dovrà essere sempre calibrata in base alla funzione renale.

Le IVU limitate alle basse vie associate a scarsa sintomatologia che si manifestino precocemente, entro i primi 4-6 mesi dal trapianto, hanno una prognosi favorevole, possono essere gestite ambulatorialmente, ma devono essere trattate con antibiotici per 10-14 giorni (Fig. 1). Un eventuale stent ureterale deve essere rimosso quanto prima e inviato per esame colturale.

Anche le infezioni non complicate con esordio oltre i 6 mesi dal trapianto hanno tendenzialmente una prognosi favorevole, non si associano a batteriemia, né influenzano la funzione del graft nel lungo termine (28) e devono essere trattate con antibiotici per almeno 5-7 giorni (Fig. 1).

Nelle infezioni associate a segni sistemici di pielonefrite è necessario invece un trattamento in regime di ricovero ospedaliero (Fig. 1). La pielonefrite del graft può associarsi a batteriemia, diffusione a distanza, peggioramento della funzione del graft, e persino morte del paziente. 


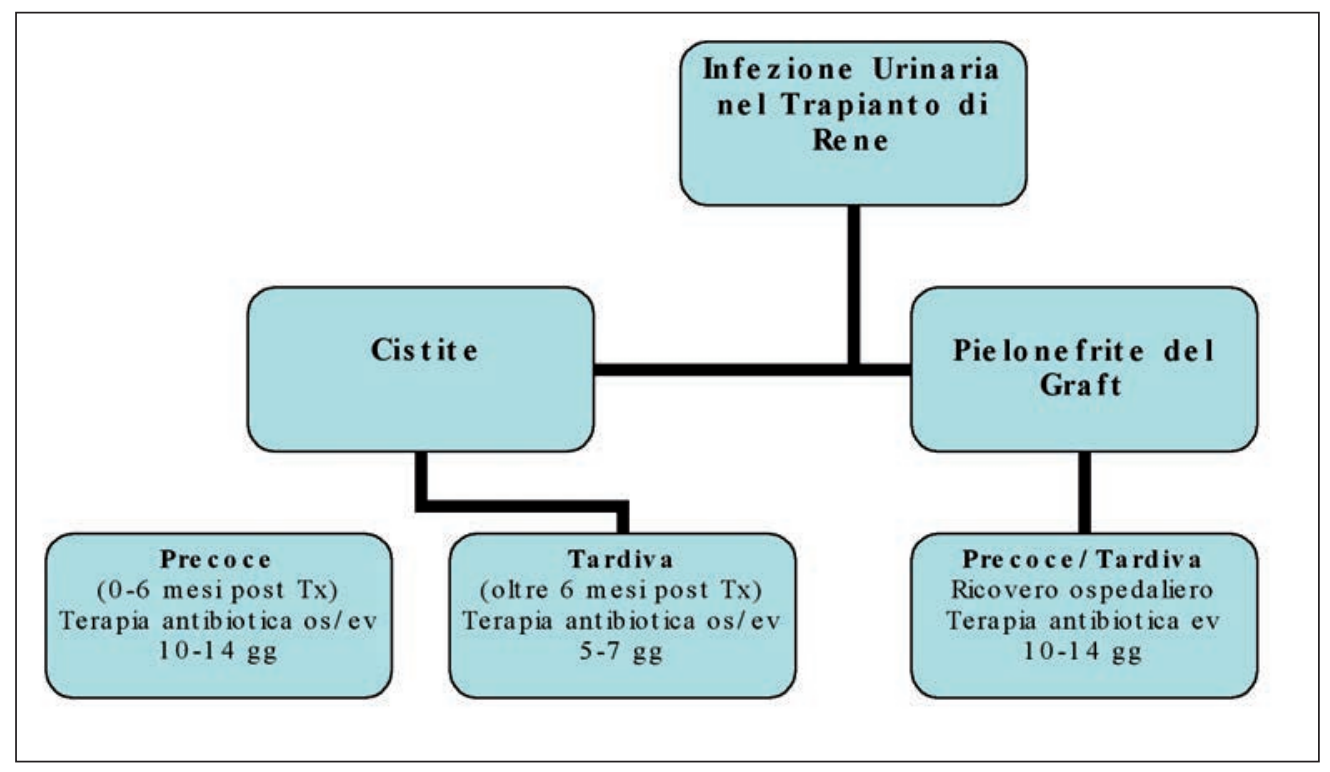

Fig. 1 - Trattamento delle IVU nel paziente adulto trapiantato di rene.

Tale forma richiede un trattamento prolungato per 2-3 settimane con idratazione e antibiotici e.v. Quando in questo contesto il quadro febbrile si protrae oltre le 48 ore dall'inizio della terapia antibiotica, oppure in presenza di segni sistemici di sepsi, è opportuno ridurre o sospendere temporaneamente l'immunosoppressione fino alla risoluzione completa dell'episodio.

\section{IVU ricorrenti}

Mancano linee guida basate sull'evidenza in merito al trattamento delle IVU ricorrenti nel trapiantato di rene, $\mathrm{i}$ risultati dei pochi studi disponibili suggeriscono l'impiego di terapie prolungate, le ricadute sono infatti generalmente la conseguenza di una terapia antibiotica troppo breve, il suggerimento è quello di protrarre il trattamento per almeno 3-6 settimane $(4,29)$. È fondamentale rivalutare la terapia immunosoppressiva, in quanto tali forme possono essere conseguenti a una eccessiva immunosoppressione e i germi responsabili possono essere ormai diventati resistenti ai comuni antibiotici in seguito alla profilassi standard (24).

Il problema maggiore nelle donne è la colonizzazione vaginale con germi uropatogeni, sono stati proposti diversi metodi a integrazione della profilassi farmacologica, tra cui l'assunzione di aumentate quantità di liquidi e la minzione frequente, in modo da garantire una adeguata clearance batterica giornaliera, la minzione prima di coricarsi, la somministrazione di steroidi intravaginali nelle donne in menopausa, l'instillazione vaginale di lactobacillus casei, che contribuisce a mantenere un $\mathrm{pH}$ uguale o inferiore a 5 (12). Tra i rimedi naturali vi è il succo di mirtillo rosso (o cranberrry juice), che sembra agire acidificando le urine e inibendo l'espressione delle fimbrie P dell'E. coli impedendo l'aderenza del batterio allurotelio (30), tuttavia il suo ruolo nella prevenzione delle IVU è ancora controverso (31).

Da una rewiew della Cochrane Library sull'uso di antibiotici nelle IVU ricorrenti in donne non gravide è emerso che l'assunzione di TMPS, nitrofurantoina, cefalessina o norfloxacina riduce il numero delle infezioni ricorrenti, tuttavia tale effetto sembra avere breve durata (32).

Nell'uomo le infezioni che tendono maggiormente a ricorrere sono le prostatiti, queste rispondono generalmente al trattamento con fluorochinoloni, cefalosporine o TMPS (29). Non vi sono indicazioni in letteratura su quale debba essere la durata della terapia nel trapiantato, tuttavia considerando la loro tendenza alla cronicizzazione, è ragionevole protrarre il trattamento per almeno 2-3 settimane, anche in questo caso è importante la guida dell'antibiogramma sull'esame colturale del secreto prostatico.

\section{IVU asintomatiche}

Non esistono linee guida sul trattamento della batteriuria asintomatica, il suo significato clinico è tuttora controverso. È stato ipotizzato che determini un danno subclinico sull'apparato urinario, la dimostrazione di elevate concentrazioni di IL6 e IL8, nelle urine di trapiantati di rene con batteriuria asintomatica rifletterebbe un processo infiammatorio in atto, che nel lungo termine sarebbe responsabile di un danno a carico del rene trapiantato (33). Alcuni autori raccomandano, nellimme- 
diato post-trapiantato, di trattare sia la batteriuria asintomatica sia quella con conta batterica bassa $(<100.000$ CFU) (29). Tale comportamento può essere giustificato in teoria dalla compresenza di fattori di rischio infettivo quali diabete, gravidanza, catetere vescicale o nefrostomico. Tuttavia non c'è evidenza in letteratura che tale strategia contribuisca a ridurre l'impatto sulla funzione del rene trapiantato (4).

Anche per la candiduria non c'è accordo sulla necessità di un suo trattamento, la frequente associazione con uso recente di antibiotici, presenza di catetere vescicale, vescica neurologica, diabete, malnutrizione, ricovero in unità di terapia intensiva, la rende un marker di gravità della situazione clinica (34). Per questo motivo è utile un suo monitoraggio. Quanto all'opportunità di una terapia in assenza di sintomi, in letteratura non esistono evidenze a supporto.

\section{Conclusioni}

Le IVU sono la complicanza più frequente nel ricevente un trapianto di rene, se non identificate e trattate in modo appropriato possono evolvere verso quadri di crescente gravità. E necessario iniziare rapidamente una terapia antibiotica empirica e avviare le indagini per cercare di identificare il focolaio infettivo e l'agente responsabile, in modo da poter orientare la terapia in modo specifico. La terapia immunosoppressiva deve essere rivalutata in funzione della gravità dellinfezione, dell'aggressività del germe responsabile, della storia immunologica del paziente. $\grave{E}$ spesso necessario ridurre o sospendere uno o più farmaci per consentire al paziente di recuperare una risposta antinfiammatoria adeguata. Pazienti con pielonefrite del graft devono essere ospedalizzati e trattati con antibiotici e.v., tale forma può associarsi a batteriemia, diffusione a distanza e condurre a peggioramento funzionale o perdita del graft, sepsi e talora morte del paziente. L'infezione può talora esordire in forma di batteriuria asintomatica, tuttavia allo stato attuale non vi sono dati in letteratura che supportino l'utilizzo della terapia antibiotica in questo stadio. La profilassi antibiotica a lungo termine, nei primi 6 mesi post-trapianto, può ridurre il rischio di IVU. Certezze in questo campo potranno emergere soltanto da studi controllati che indaghino la durata ottimale della profilassi nel lungo termine e l'incidenza di infezioni ricorrenti alla sospensione della profilassi.

\section{Riassunto}

Le infezioni delle vie urinarie (IVU) sono la complicanza più frequente nel ricevente un trapianto di rene, se non identificate e trattate in modo appropriato possono evolvere verso quadri di crescente gravità. Una volta fatta la diagnosi di IVU è necessario iniziare rapidamente una terapia antibiotica empirica e avviare le indagini per cercare di identificare focolaio infettivo e agente responsabile, in modo da poter orientare la terapia in modo specifico. La terapia immunosoppressiva deve essere rivalutata in funzione della gravità dell'infezione, dell'aggressività del germe responsabile, della storia immunologica del paziente. È spesso necessario ridurre o sospendere uno o più farmaci per consentire al paziente di recuperare una risposta antinfiammatoria adeguata. Pazienti con pielonefrite del graft devono essere ospedalizzati e trattati con antibiotici e.v., tale forma può associarsi a batteriemia, diffusione a distanza e condurre a peggioramento funzionale o perdita del graft, sepsi e talora morte del paziente. Sono necessari studi controllati che permettano di definire meglio alcuni aspetti delle IVU nel trapiantato di rene, in particolare la durata ottimale della profilassi nel lungo termine e l'incidenza di IVU ricorrenti alla sospensione della profilassi.

\section{Indirizzo degli Autori: \\ Jacopo Romagnoli, $\mathrm{MD}, \mathrm{PhD}$ \\ Istituto di Clinica Chirurgica \\ Policlinico "A. Gemelli" \\ Università Cattolica del Sacro Cuore \\ Policlinico Gemelli \\ Largo Gemelli 8 \\ 00168 Roma \\ jromagnoli@rm.unicatt.it}

\section{Bibliografia}

1. Schmaldienst S, Dittrich E, Horl WH. Urinary tract infections after renal transplantation. Curr Opin Urol 2002; 12: 125-30.

2. Alangaden GJ, Thyagarajan R, Gruber SA, et al. Infectious complicationsafter kidney transplantation: current epidemiology and associated risk factors. Clin 
Transplant 2006; 20: 401-9.

3. Dantas SR, Kuboyama RH, Mazzali M, et al. Nosocomial infections in renal transplant patients: risk factors and treatment implications associated with urinary tract and surgical site infections. J Hosp Infect 2006; 63: 117-23.

4. De Souza RM, Olsburgh J. Urinary tract infection in the renal transplant patient. Nat Clin Pract Nephrol 2008; 4: 252-64.

5. Saemann M, Horl WH. Urinary tract infection in renal transplant recipients. Eur J Clin Invest 2008; 38 (Suppl 2): S58-65.

6. Chuang P, Parikh CR, Langone A. Urinary tract infections after renal transplantation: a retrospective review at two US transplant centers. Clin Transplant 2005; 19: 230-5.

7. Pellé G, Vimont S, Levy PP, et al. Acute pyelonephritis represents a risk factor impairing long-term kidney graft function. Am J Transplant 2007; 7: 899-907.

8. Abbott KC, Swanson SJ, Richter ER, et al. Late urinary tract infection after renal transplantation in the United States. Am J Kidney Dis 2004; 44: 353-62.

9. Krcmery S, Hromec J, Gábrisová $Z$, et al. Fungal urinary tract infections in patients at risk. Int J Antimicrob Agents1999; 11: 289-91.

10. Schmiemann G, Kniehl E, Gebhardt K, et al. The Diagnosis of Urinary Tract Infection. A Systematic Review. Dtsch Arztebl Int 2010; 107(21): 361-7.

11. Rivera-Sanchez R, Delgado-Ochoa D, Flores-Paz RR, et al. Prospective study of urinary tract infection surveillance after kidney transplantation. BMC Infect Dis 2010; 10: 245-51.

12. Franco AVM. Recurrent Urinary tract infections. Best Pract Res Clin Obstet Gynaecol 2005;19: 861-73.

13. Boyko EJ, Fihn SD, Scholes D, Abraham L, Monsey B. Risk of urinary tract infection and asymptomatic bacteriuria among diabetic and nondiabetic postmenopausal women. Am J Epidemiol 2005; 161: 557-64.

14. Wilson Ch, Bhatti AA, Rix DA, et al. Routine intraoperative ureteric stenting for kidney transplant recipients. Cochrane Database Syst Rev 2005; 4: CD004925.

15. Stamm WE, Hooton TM. Management of urinary tract infections in adults. N Engl J Med 1993; 329: 1328-34.

16. Nicolle LE. Urinary tract infection: traditional pharmacologic therapies. Am J Med 2002;113: 35-44.

17. Ronald AR, Harding GKM. Complicated urinary infections. Infectious Disease Clinic of North America 1997; 11: 583-92.

18. Svanborg C, Goldaly G. Bacterial virulence in urinary tract infection. Infectious Disease Clinic of North America 1997; 11: 513-29.

19. Kucheria R, Sheerin NS, Dasgupta P, et al. Urinary tract infections: advances and new therapies. BJU Int 2004;
93: 690-91.

20. Kuback BM, Maree CL, Pegues DA, Hwang. In: G.M. Danovitch, Handbook of Kidney Transplantation, $4^{\text {th }}$ edition, Philadelphia, Lippincott Williams e Wilkins, 2005; 279-333.

21. Hirsch HH. Polyomavirus BK nephropathy:a re-emerging complication in renal transplantation. Am J Transplant 2002 2: 25-30.

22. Stapleton A. Prevention of recurrent urinary tract infections in women. Lancet 1999; 353: 7-8.

23. Johnson JR. Microbial virulence determinants and the pathogenesis of urinary tract infection. Infectious Disease Clinics of North America 2003; 17: 261-78.

24. Blango Mc, Mulvery MA. Persistence of uropathogenic E. Coli in the face of multiple antibiotics. Antimicrob Agents Chemother 2010; 54: 1855-63.

25. Banyai-Falger S, Mayer U, Susani M, et al. High incidence of nephrogenic adenoma of the bladder after renal transplantation. Transplantation 1998; 65: 511-4.

26. Naber KG, Hofstetter AG, Brühl P, et al. Guidelines for the perioperative prophylaxis in urological interventions of the urinary and male genital tract. Int J Antimicrob Agents 2001;17: 321-6.

27. Fox BC, Sollinger HW, Belzer FO, et al. A prospective, randomized,double-blind study of trimethoprimsulfamethoxazole for prophylaxis of infection in renal transplantation: Clinical efficacy, absorption of trimethoprim-sulfamethoxazole, effects on the microflora, and the cost-benefit of prophylaxis. Am J Med 1990; 89: 255-74.

28. Kidney Disease: Improving Global Outcomes (KDIGO) Transplant Work Group. KDIGO clinical practice guideline for the care of kidney transplant recipients. Am J Transplant 2009; 9 (Suppl 3): S1-155.

29. Common errors in diagnosis and management of urinary tract infections. II: clinical magement. Franz M, Horl phrol Dial Transplant 1999; 14: 2754-62.

30. Avornj, Monane M, Gurwitz J, et al. Reduction of bacteruria and pyuria after ignestion of cranberry juice. JAMA 1994; 271: 52-53.

31. Jepson RG, Mihaljevic L, Craig J. Cranberries for preventing urinary tract infections. Cochrane Database of Systematic Reviews 2008;1: CD001321.

32. Albert X, Huertas I, Pereiro I, et al. Antibiotics for preventing urinary tract infections in nonpregnant women. Cochrane Collaboration 2005; 2: 1-54.

33. Ciszek M, Paczek L, Bartłomiejczyk I, et al. Urine cytokines profile in renal transplant patients with asymptomatic bacteriuria. Transplantation 2006; 81: 1653-7.

34. Safdar N, Slattery WR, Knasinski V, et al. Clin Infect Disease 2005; 40: 1413-21. 